lam Vita Jabour

\title{
O Impacto de Atributos Estruturais na Identificação de Tabelas e Listas em Documentos HTML
}

Dissertação apresentada como requisito parcial para obtenção do grau de Mestre pelo Programa de Pós-graduação em Informática do Departamento de Informática da PUC-Rio

Orientador : Prof. Eduardo Sany Laber Co-Orientador: Prof. Raúl Pierre Rentería 


\title{
O Impacto de Atributos Estruturais na Identificação de Tabelas e Listas em Documentos HTML
}

Dissertação apresentada como requisito parcial para obtenção do grau de Mestre pelo Programa de Pós-graduação em Informática do Departamento de Informática do Centro Técnico Científico da PUC-Rio. Aprovada pela Comissão Examinadora abaixo assinada.

\author{
Prof. Eduardo Sany Laber \\ Orientador \\ Departamento de Informática - PUC-Rio \\ Prof. Raúl Pierre Rentería \\ Co-Orientador \\ Departamento de Informática - PUC-Rio \\ Prof. Ruy Luiz Milidiú \\ Departamento de Informática - PUC-Rio
}

Prof. Rogério Ferreira Rodrigues

Departamento de Informática - PUC-Rio

Prof. Alexandre Plastino de Carvalho Departamento de Ciência da Computação - UFF

Prof. José Eugenio Leal Coordenador Setorial do Centro Técnico Científico - PUC-Rio 
Todos os direitos reservados. É proibida a reprodução total ou parcial do trabalho sem autorização da universidade, do autor e do orientador.

\section{Iam Vita Jabour}

Graduou-se em Bacharelado em Informática na PUC-Rio.

Ficha Catalográfica

Jabour, lam

O Impacto de Atributos Estruturais na Identificação de Tabelas e Listas em Documentos HTML / lam Vita Jabour; orientador: Eduardo Sany Laber; co-orientador: Raúl Pierre Rentería. - Rio de Janeiro : PUC-Rio, Departamento de Informática, 2010.

$$
\text { v., } 64 \text { f: il. ; } 29,7 \mathrm{~cm}
$$

1. Dissertação (mestrado) - Pontifícia Universidade Católica do Rio de Janeiro, Departamento de Informática.

Inclui referências bibliográficas.

1. Informática - Tese. 2. extração de informação. 3. extração de tabelas. 4. extração de listas. 5. segmentação de documentos HTML. 6. isomorfismo em árvore. I. Laber, Eduardo. II. Rentería, Raúl. III. Pontifícia Universidade Católica do Rio de Janeiro. Departamento de Informática. IV. Título. 


\section{Agradecimentos}

Aos meus pais, por todo o apoio e carinho.

A minhas irmãs, por serem pessoas especiais em minha vida.

Aos orientadores Prof. Eduardo Laber e Prof. Raúl Rentería, pelo apoio e incentivo, proporcionando uma orientação exemplar e estando ambos sempre disponíveis e presentes durante o desenvolvimento desta dissertação.

Aos professores da banca examinadora, pelas criticas e sugestões que contribuíram para o aprimoramento deste trabalho.

Ao $\mathrm{CNPq}$ e à PUC-Rio, pelos auxílios concedidos, sem os quais este trabalho não poderia ter sido realizado.

A todos os meus colegas da pós-graduação, que participaram contribuindo com sugestões e incentivos. 


\section{Resumo}

Jabour, Iam; Laber, Eduardo; Rentería, Raúl. O Impacto de Atributos Estruturais na Identificação de Tabelas e Listas em Documentos HTML. Rio de Janeiro, 2010. 64p. Dissertação de Mestrado Departamento de Informática, Pontifícia Universidade Católica do Rio de Janeiro.

A segmentação de documentos HTML tem sido essencial para as tarefas de extração de informações, como mostram vários estudos na área. Nesta dissertação investigamos a relação entre o documento HTML e sua representação visual, mostrando como esta ligação ajuda na abordagem estrutural para a identificação de segmentos. Também investigamos como utilizar algoritmos de distância de edição em árvores para encontrar padrões na árvore DOM, tornando possível resolver duas tarefas de identificação de segmentos. A primeira tarefa é a identificação de tabelas genuínas, aonde foi obtido $90,40 \%$ de $F_{1}$ utilizando o corpus fornecido por (Wang e Hu, 2002). Mostramos através de um estudo experimental que este resultado é competitivo com os melhores resultados da área. A segunda tarefa que consideramos é a identificação de listas de produtos em sites de comércio eletrônico, nessa obtivemos 94,95\% de $F_{1}$ utilizando um corpus com 1114 documentos HTML, criado a partir de 8 sites. Concluímos que os algoritmos de similaridade estrutural ajudam na resolução de ambas às tarefas e acreditamos que possam ajudar na identificação de outros tipos de segmentos.

\section{Palavras-chave}

extração de informação; extração de tabelas; extração de listas; segmentação de documentos HTML; isomorfismo em árvore; 


\section{Abstract}

Jabour, Iam; Laber, Eduardo(Advisor); Rentería, Raúl. The Impact of Structural Attributes to Identify Tables and Lists in HTML Documents. Rio de Janeiro, 2010. 64p. MSc Dissertation — Departamento de Informática, Pontifícia Universidade Católica do Rio de Janeiro.

The segmentation of HTML documents has been essential to information extraction tasks, as showed by several works in this area. This paper studies the link between an HTML document and its visual representation to show how it helps segments identification using a structural approach. For this, we investigate how tree edit distance algorithms can find structural similarities in a DOM tree, using two tasks to execute our experiments. The first one is the identification of genuine tables where we obtained a $90.40 \% F_{1}$ score using the corpus provided by (Wang e Hu, 2002). We show through an experimental study that this result is competitive with the best results in the area. The second task studied is the identification of product listings in e-commerce sites. Here we get a $94.95 \% F_{1}$ score using a corpus with 1114 HTML documents from 8 distinct sites. We conclude that algorithms to calculate trees similarity provide competitive results for both tasks, making them also good candidates to identify other types of segments.

\section{Keywords}

information extraction; table extraction; list extraction; webpage segmentation; isomorphism; 


\section{Sumário}

1 Introdução $\quad 10$

$\begin{array}{lll}1.1 & \text { Definição do problema } & 11\end{array}$

1.2 Contribuições 13

$\begin{array}{lll}1.3 & \text { Organização da dissertação } & 15\end{array}$

2 Conceitos básicos $\quad 16$

2.1 A linguagem HTML 16

2.2 Formas de visualização de um documento HTML 19

2.3 Document Object Model (DOM) 22

3 Abordagem $\quad 24$

3.1 Algoritmos de similaridade em árvore 24

3.2 O ambiente de experimentação 33

4 Identificação de tabelas $\quad 37$

4.1 Trabalhos existentes 40

4.2 Métricas de avaliação 42

4.3 Corpus de exploração 43

4.4 Abordagem proposta 43

$5 \quad$ Extraindo listas de produtos em sites de comércio eletrônico $\quad 51$

5.1 Trabalhos existentes $\quad 52$

5.2 Corpus de exploração $\quad 54$

5.3 Métrica $\quad 55$

$\begin{array}{lll}5.4 & \text { Abordagem proposta } & 55\end{array}$

6 Conclusões $\quad 60$

$\begin{array}{ll}\text { Referências Bibliográficas } & 62\end{array}$ 


\section{Lista de figuras}

1.1 Exemplo de segmentos retirado do site do UOL. 11

1.2 Exemplo de identificação de um produto do site bestbuy.com 12

2.1 Fluxo principal do WebKit 19

2.2 Comparação entre o documento HTML e sua árvore DOM 22

2.3 Comparação entre a visualização do documento em um navegador e sua árvore DOM 23

3.1 Exemplo de dois conjuntos generalizadores de tamanho 5

3.2 Exemplo de data regions (Regions), retirado de (Liu et. al., 2003) 26

3.3 Ilustração de dois nós generalizadores de tamanho $5 \quad 28$

3.4 Ilustração de duas árvores A e B retirado de (Yang, 1991) 29

3.5 Ilustração da tabela final do procedimento (C) e do cálculo da primeiro nível de mapeamento (D) retirado de (Yang, 1991) 30

3.6 Procedimento Simple Tree Matching 31

3.7 Procedimento Casamento Simples 31

3.8 Exemplo de estrutura que utiliza um conjunto generalizador de tamanho 2 para apresentar os itens 33

3.9 Diagrama de classes da ferramenta 34

3.10 Diagrama de sequência da aplicação Benchmark 36

4.1 Tabela de difícil compreensão retirada de (Tengli et. al., 2004) 37

4.2 Tabela não genuína com a árvore HTML correspondente à direita 39

4.3 Tabela genuína com a árvore HTML correspondente a direita 40

5.1 Exemplo de uma lista de produtos do site bestbuy.com 52

5.2 Exemplo de uma lista de produtos do site americanas.com 53 


\section{Lista de tabelas}

4.1 Classificações possíveis de uma tabela para o cálculo das métricas 42

4.2 Resultados sobre o conjunto de treino sem utilizar técnicas específicas para tabelas

4.3 Resultados no conjunto de treino utilizando a função razão de linhas (RL) com as técnicas de semelhança de estrutura

4.4 Resultado das técnicas sobre o conjunto de teste

4.5 Resultados de aprendizado de máquina com validação cruzada sobre o corpus completo

4.6 Comparação dos resultados de identificação de tabelas genuínas com os trabalhos relacionados

4.7 Tempo de processamento em segundos dos 1393 documentos com os algoritmos propostos

5.1 Classificações possíveis de uma tabela para o cálculo das métricas 55

5.2 Testes iniciais com 24 documentos do corpus de treino

5.3 Escolha do melhor método com todos os documentos do corpus de treino

5.4 Resultados no corpus de teste

5.5 Regras específicas sobre o corpus de treino

5.6 Resultado $F_{1}$ das regras específicas no corpus de teste 